\title{
Optical Properties of DMA- $\pi$-DCV Derivatives: A Theoretical Inspection under the DFT Microscope
}

\author{
Joaquín Calbo \\ Instituto de Ciencia Molecular, Universidad de Valencia, 46890 Paterna, Spain \\ Correspondence should be addressed to Joaquín Calbo; joaquin.calbo@uv.es
}

Received 17 August 2016; Accepted 4 October 2016

Academic Editor: Jau-Wern Chiou

Copyright (C) 2016 Joaquín Calbo. This is an open access article distributed under the Creative Commons Attribution License, which permits unrestricted use, distribution, and reproduction in any medium, provided the original work is properly cited.

\begin{abstract}
The optical properties of a series of donor-acceptor N,N-dimethylaniline- $\pi$-dicyanovinylene (DMA- $\pi$-DCV) chromophores have been investigated under the density functional theory framework. Focus has been made on the low-lying charge-transfer (CT) electronic transitions for which experimental data is available. The effect of the $\pi$-conjugated bridge length and type was analysed between the families of oligoene and oligoyne derivatives of increasing size. Theoretical calculations demonstrate that the ethylene bridge is a better $\pi$-communicator and allows for more delocalized frontier molecular orbitals compared to the acetylene spacer. The $\Lambda$ diagnostic test allowed rationalization of the orbital spatial overlap in the main CT excitations. The performance of different density functional rungs was assessed in the prediction of the lowest-lying CT electronic transition. Surprisingly, most modern long-range corrected functionals demonstrated to provide among the largest errors, whereas hybrid functionals showed the best performance. Solvatochromism was confirmed in both oligoene and oligoyne compounds. A donor-acceptor-donor triad based on tetrathiafulvalene was utilised as a test system for the prediction of its two CT bands of different nature, energy, and intensity. The hybrid PBE0 (or a similar hybrid analogue) consolidates as the best choice for the prediction of CT excitations in the DMA- $\pi$-DCV push-pull family.
\end{abstract}

\section{Introduction}

Organic chromophores have attracted increasing attention in a variety of fields such as molecular electronics and optoelectronics due to their high degree of $\pi$-conjugation, giving them unique electronic and optical properties [1-4]. Donoracceptor (D-A) conjugated organic molecules constitute one of the most promising derivatives in this family, so-called push-pull systems, with particular interest in their secondand third-order nonlinear optical properties [5]. In a donoracceptor compound, an electron-rich moiety (donor) gives electronic density to the electron-poor fragment (acceptor). The charge transfer occurring in these systems is highly dependent on the nature (strength) of the donor and acceptor moieties, as well as on the $\pi$-conjugation efficiency and length of the bridge connecting both parts. Still, however, the amount of charge transfer in the ground state is far below the approximately one-electron transfer that proceeds upon photoirradiation. In push-pull systems, the highestoccupied molecular orbital (HOMO) is usually placed in the donor moiety, whereas the lowest-unoccupied molecular orbital (LUMO) is found in the acceptor fragment. The photoexcitation of D-A entities therefore produces a lowlying charge-transfer electronic excitation described by the one-electron promotion from the HOMO to the LUMO, where energy absorption highly depends on the linear D-A conjugation pathways $[6,7]$. Stronger D-A coupling across shorter $\pi$-conjugated spacers leads to higher-energy absorptions, whereas weaker D-A coupling across longer spacers results in lower HOMO-LUMO gaps [8-10].

To date, one of the most used donor architectures in pushpull systems is the N,N-dimethylaniline (DMA) structure, which has allowed the generation of a vast number of D-A systems, both planar and nonplanar $[8,11,12]$. Whereas planar push-pull chromophores featuring intramolecular chargetransfer (CT) interactions have been extensively studied, only a limited number of nonplanar low-molecular-weight donoracceptor chromophores have been reported $[13,14]$. On the other hand, the dicyanovinylene (DCV) group has become one of the most employed electron-accepting architectures in 


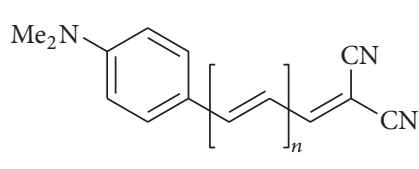

$n=1-3$

oe- $n$

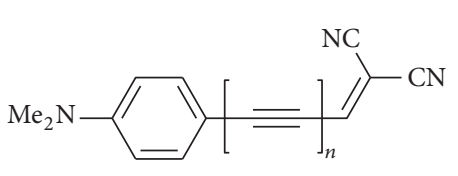

$n=1-3$

oy- $n$

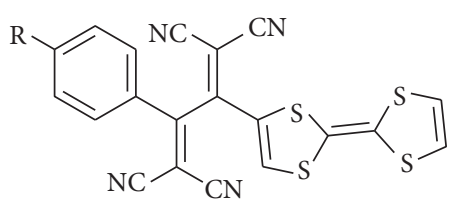

$\mathrm{R}=\mathrm{H}, \mathrm{NMe}_{2}$ TTF-TCB-R

Scheme 1: List of compounds under study: the two families of DMA- $\pi$-DCV derivatives (oligoenes with - $\mathrm{CH}=\mathrm{CH}-\mathrm{bridge}$, left; oligoynes with $-\mathrm{C} \equiv \mathrm{C}$ - bridge, centre) and the TTF-based push-pull chromophores (right).

the generation of efficient push-pull systems to be exploited in organic electronics [15-18]. The combination of both the DMA and the DCV moieties led to the synthesis of a bunch of $\mathrm{D}$-A compounds with tuneable properties à la carte $[8,11$, 12, 19-24].

Particularly interesting is the modulation of the bridge connecting the donor and the acceptor moieties. Recently, two types of $\pi$-conjugated bridges have been employed as spacers in the construction of push-pull chromophores to tune the optical and electronic properties of D-A-substituted oligoynes (spacer: $-\mathrm{C} \equiv \mathrm{C}-$ ) and oligoenes (spacer: $-\mathrm{CH}=\mathrm{CH}-$ ) $[12,25-27]$. Oligoenes based on DMA- $\pi$-DCV (oe- $n$ in Scheme 1) demonstrated having a better ability to produce good homogeneous solutions for the nonlinear optical measurements, and showed a clear increase in thirdorder polarizability with the size of the spacer. Oligoynebased derivatives (oy- $n$, Scheme 1) were much less capable of producing a good homogeneous solution with signs of decomposition for the larger molecules, and the third-order polarizability was already saturated at the diyne compound [12]. These systems showed a clear, intense, low-lying CT band recorded in the $450-600 \mathrm{~nm}$ range, whose position and intensity can be modulated by the extension of the $\pi$ conjugated bridge.

On the other hand, the tetrathiafulvalene (TTF) and its derivatives constitute a widely used electron-donor fragment in the generation of nonplanar chromophores [28-33]. A TTF-based derivative was recently synthesized possessing a nonplanar shape and appealing optical properties (TTFTCB-NMe $e_{2}$ in Scheme 1) [11]. The presence of two electrondonor moieties in this system (TTF and DMA), together with an electron-accepting tetracyanobutadiene (TCB) as a bridge, led to the presence of two intense, low-lying CT states in the electronic absorption spectrum. The higher-energy transition located in the $450-500 \mathrm{~nm}$ region was assigned to the DMA $\rightarrow$ TCB electronic excitation, whereas the lowerenergy band recorded at $729 \mathrm{~nm}$ was ascribed to the TTF $\rightarrow$ TCB promotion. This system constitutes a great example containing two clearly characterized low-lying CT bands with different nature, energy, and intensity.

From the experimental point of view, complications in the synthesis paths, together with solubility issues and the ubiquitous environmental impact effect, point toward a desirable computational screening of a large list of DA derivatives, to then focus on the synthesis of the most promising candidates with optimal optical and electronic properties [34]. To reach that goal, available theoretical approaches must predict accurately the desired properties, for example, the electronic absorption spectrum in push-push systems, in an affordable computational cost-accuracy ratio. In this line, density functional theory (DFT) has marked a step forward in the design of chromophores based on the rationalization of valuable structure-property relationships [28-33, 35].

Although a widely recognised failure in DFT is the prediction of accurate energetics for the low-energy charge-transfer bands [36, 37], much effort has been devoted to palliate this shortcoming by the introduction of long-range corrected density functionals that include a variable amount of exact Hartree-Fock (HF) exchange to the functional expression as a function of distance [38]. The better description of the CT excitations generally obtained by these long-range corrected functionals is counterbalanced by a larger computational cost, accompanied with a more complicated description of the electronic excitations as well as a poorer prediction of the $\pi-\pi^{*}$ transitions in common organic chromophores. Additionally, a diagnostic test (so-called $\Lambda$ ) permits the identification of CT excitations, in which conventional density functionals should be avoided, by the estimation of the spatial overlap between the orbital pairs involved in the electronic transition of interest [39].

In this work, we present a theoretical study on the optical features of a list of chromophores containing DMA as a donor group and DCV as an acceptor moiety. The effect of the type and length of the bridge (ethylene versus acetylene) on the low-lying charge-transfer excitation is analysed by performing TDDFT calculations on the oligoene and oligoyne families and is related to the HOMO-LUMO gap. The performance of the different density functional rungs has been assessed, and the spatial overlap between the orbitals involved in the excitation of interest is evaluated through the calculation of the $\Lambda$ diagnostic test. The percentage of the exact Hartree-Fock exchange in the formulation of the functional is found to be key for the accurate prediction of the low-lying CT excitations by hybrid functionals. The composite chromophore TTF-TCB-NMe 2 containing the donor tetrathiafulvalene moiety was used as a test system possessing two charge-transfer electronic bands of different nature, energy, and intensity.

\section{Materials and Methods}

Preliminary exploration of the geometric, electronic, and optical properties of the list of compounds under study was 


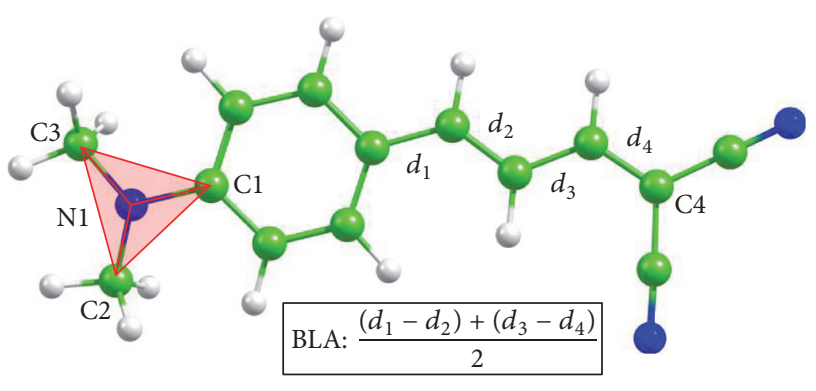

SCHEME 2

carried out by performing geometry optimizations under the density functional theory (DFT) framework by making use of the hybrid exchange-correlation B3LYP functional [40], in combination with Pople's $6-31 \mathrm{G}^{* *}$ basis set [41] and in gas phase. The lowest-lying singlet excited states were computed at the same level of theory by means of the time-dependent DFT approach [42-44]. The lowest-lying 20 singlet excited states were computed for both oligoene and oligoyne derivatives.

Effects on the geometry were considered by optimizing all the structures under the $a b$ initio MP2 level of theory [45] using the $6-31 G^{* *}$ basis set. Effects of the nitrogen pyramidalization and of the bond length alternation (BLA) were analysed by measuring characteristic dihedral angles and bond distances. The bond length alternation index was measured as the difference between single and double/triple bonds in the bridge for both oligoenes and oligoynes divided by the number of groupings (see Scheme 2).

The performance of a variety of density functionals on the optical properties of the DMA- $\pi$-DCV derivatives was carried out by employing the pure-GGA PBE [46] and BLYP $[47,48]$ functionals, the hybrid B3LYP [40] and PBE0 [49], the hybrid meta-GGA M06-2X [50], the range-separated HSE03 [51], and the long-range corrected CAM-B3LYP [52] and wB97XD [53]. The effect of the basis set was analysed by computing the lowest-lying CT transition using the B3LYP functional combined with the smaller 6-31G [54] and the bigger $6-311+\mathrm{G}^{* *}[55]$ basis sets for the oligoene family. Small energy deviations were found within an acceptable range of $\pm 0.1 \mathrm{eV}$. Solvent effects were taken into account by means of the self-consistent reaction field (SCRF) using both the polarizable continuum model (PCM) [56] and the solvent model based on solute electron density (SMD) [57] and dichloromethane as a solvent.

The $\Lambda$ diagnostic test was calculated as described in [39], by measuring the spatial overlap between the occupied orbital $\varphi_{i}$ and the virtual orbital $\varphi_{a}$ that contribute to the relevant singlet excited state according to

$$
O_{i a}=\left\langle\left|\varphi_{i}\right||| \varphi_{a} \mid\right\rangle=\int\left|\varphi_{i}(\mathbf{r})\right|\left|\varphi_{a}(\mathbf{r})\right| d \mathbf{r}
$$

The spatial overlap between two orbitals was calculated by means of the Gabedit 2.4.8 software [58]. Many occupiedvirtual pairs may contribute to a given TDDFT excitation, and the contribution from each pair can be measured by

$$
\kappa_{i a}=X_{i a}+Y_{i a}
$$

where $X_{i a}$ and $Y_{i a}$ are elements of the solution vectors of the TDDFT generalized eigenvalue problem. A measure of spatial overlap for a given excitation can therefore be obtained by weighting each inner product $O_{i a}$ by some function $f\left(\kappa_{i a}\right)$ and summing over all occupied-virtual pairs, suitably normalized. The spatial overlap in a given excitation is therefore usually measured according to the expression

$$
\Lambda=\frac{\sum_{i, a} \kappa_{i a}^{2} O_{i a}}{\sum_{i, a} \kappa_{i a}^{2}}
$$

which takes the value $0 \leq \Lambda \leq 1$. A small value of $\Lambda$ signifies a long-range excitation, whereas a large value signifies a shortrange excitation. In our case, we took only the spatial overlap between the pair of orbitals that contribute the most on the low-lying singlet excitation $(>90 \%)$.

The effect of the amount of exact Hartree-Fock (HF) exchange in the prediction of low-lying singlet excited states of charge-transfer nature was analysed on the families of functionals PBE and BLYP. Pure-GGA PBE and BLYP functionals contain $0 \%$ of exact HF exchange, whose amount was consistently increased up to $50 \%$ according to each hybrid functional expression. In the case of the BLYP family, we took the expression for the B3LYP functional of the form

$$
\begin{aligned}
E_{x c}= & E_{x}^{\mathrm{LDA}}+a_{0}\left(E_{x}^{\mathrm{HF}}-E_{x}^{\mathrm{LDA}}\right)+a_{x}\left(E_{x}^{\mathrm{GGA}}-E_{x}^{\mathrm{LDA}}\right) \\
& +E_{c}^{\mathrm{LDA}}+a_{c}\left(E_{c}^{\mathrm{GGA}}-E_{c}^{\mathrm{LDA}}\right),
\end{aligned}
$$

where $a_{0}=0.20, a_{x}=0.72$, and $a_{c}=0.81, E_{x}^{\mathrm{LDA}}, E_{x}^{\mathrm{HF}}$, and $E_{x}^{\mathrm{GGA}}$ are the local, Hartree-Fock exact, and GGA exchange, and $E_{c}^{\mathrm{LDA}}$ and $E_{c}^{\mathrm{GGA}}$ are the local and GGA correlation, respectively. In this case, the parameter $a_{0}$ was modified from 0.00 (BLYP) to 0.50 (BHandHLYP) by steps of 0.10 , going through 0.20 (B3LYP).

For the PBE family, the PBE0 formula is set to

$$
E_{x c}=a_{0} E_{x}^{\mathrm{HF}}+\left(1-a_{0}\right) E_{x}^{\mathrm{PBE}}+E_{c}^{\mathrm{PBE}},
$$

where $a_{0}=0.25$ and $E_{x}^{\mathrm{PBE}}$ and $E_{c}^{\mathrm{PBE}}$ are the exchange and correlation parts of the $\mathrm{PBE}$ functional, respectively. The parameter $a_{0}$ was modified from 0.00 (PBE) to 0.50 by steps of 0.10 , going through 0.25 (PBE0).

The solvatochromism in the two families of compounds under study was assessed by performing single-point calculations at the gas-phase B3LYP geometry on the oligoene and oligoyne derivatives under the PCM using methylcyclohexane $(\mathrm{MCH}, \varepsilon=2.0)$, tetrahydrofuran (THF, $\varepsilon=7.4$ ), dichloromethane (DCM, $\varepsilon=8.9)$, methanol $(\mathrm{MeOH}, \varepsilon=$ 32.6), and $\mathrm{N}, \mathrm{N}$-dimethylformamide (DMF, $\varepsilon=37.2$ ).

The minimum-energy structure of the TTF-TCB-R derivatives was calculated by using the $\mathrm{B} 3 \mathrm{LYP} / 6-31 \mathrm{G}^{* *}$ level of theory in gas phase. The lowest-lying singlet excited states were computed at the TD-DFT level using the list of functionals previously assessed, under the solvent effects using PCM 
(dichloromethane as a solvent). Only the results for representative functionals of pure-GGA (BLYP), hybrid (PBE0), and long-range corrected (wB97XD) rungs are discussed. The $\Lambda$ diagnostic test was calculated as previously described by measuring the spatial overlap of the occupied-virtual orbital pair mainly involved in the electronic transition of interest.

\section{Results and Discussion}

3.1. Ground State Electronic Structure. The families of oligoene (oe- $n$ ) and oligoyne (oy- $n$ ) derivatives (Scheme 1 ) were fully optimized at the B3LYP/6-31G ${ }^{* *}$ level of theory in gas phase. All the systems present a coplanar structure in which the N,N-dimethylaniline (DMA), the $\pi$-conjugated bridge, and the dicyanovinylene (DCV) moiety remain in the same plane. The electronic structure of these compounds was calculated at the same level of theory, and the frontier molecular orbital diagrams for oe-1 and oy-1, as representative examples of the two families, are depicted in Figure 1. The highestoccupied molecular orbital (HOMO) and HOMO -1 are $\pi$ orbitals mostly delocalized along the whole structure, with certain contribution of the $\mathrm{N}$ lone pair (see Figure 1 for the topology of the MOs for oy-1). The lowest-unoccupied molecular orbital (LUMO) is also a $\pi$-type orbital, which spreads along the $\pi$-structure of the system. Interestingly, the HOMO tends to localize in the donor DMA moiety, whereas the LUMO is mainly located on the acceptor DCV fragment. The electronic promotion of one electron from the HOMO (in the donor) to the LUMO (in the acceptor) leads to the so-called charge-transfer (CT) electronic transition, which is commonly found in the low-energy range. The LUMO + 1 orbital is computed to be highly localized in the benzene moiety; indeed, it corresponds to one of the LUMOs of a benzene molecule (Figure 1). The position of this orbital with respect to higher LUMOs varies upon increasing the $\pi$-conjugated bridge. For instance, in the eo- $n$ family, the LUMO +1 of oe- 2 and oe- 3 is computed to be a $\pi$-orbital highly delocalized along the whole system, as well as that found for oy- 2 and oy- 3 in the oy- $n$ family.

A rough measure of the position of the CT electronic transition is the HOMO-LUMO gap. Figure 2 displays the HOMO-LUMO energy gap evolution as a function of the bridge size. Upon increasing the ethylene bridge length going from oe- 1 to oe-3, the HOMO consistently increases in energy from -5.49 to $-5.17 \mathrm{eV}$, whereas the LUMO decreases from -2.52 to $-2.79 \mathrm{eV}$. As a consequence, the $\mathrm{H}-\mathrm{L}$ gap is reduced upon increasing the bridge size, and for oe- 3 it is computed to be the smallest in the series at $2.38 \mathrm{eV}$. This trend agrees with the experimental evolution of the lowest-lying absorption band, which is recorded to move from $2.77 \mathrm{eV}$ in oe- 1 to $2.30 \mathrm{eV}$ in oe-3 [12]. Moving to the oligoyne family, a similar behaviour is predicted for the LUMO energy. However, an increase in the $\pi$-conjugation of the bridge is not translated into an increase in the HOMO energy, which is computed to be around $5.6 \mathrm{eV}$ along the whole family (Figure 2). The nature of the acetylene bridge implies a reduced number of antibonding interactions upon increasing the bridge size compared to the ethylene bridge. Nevertheless, the H-L gap

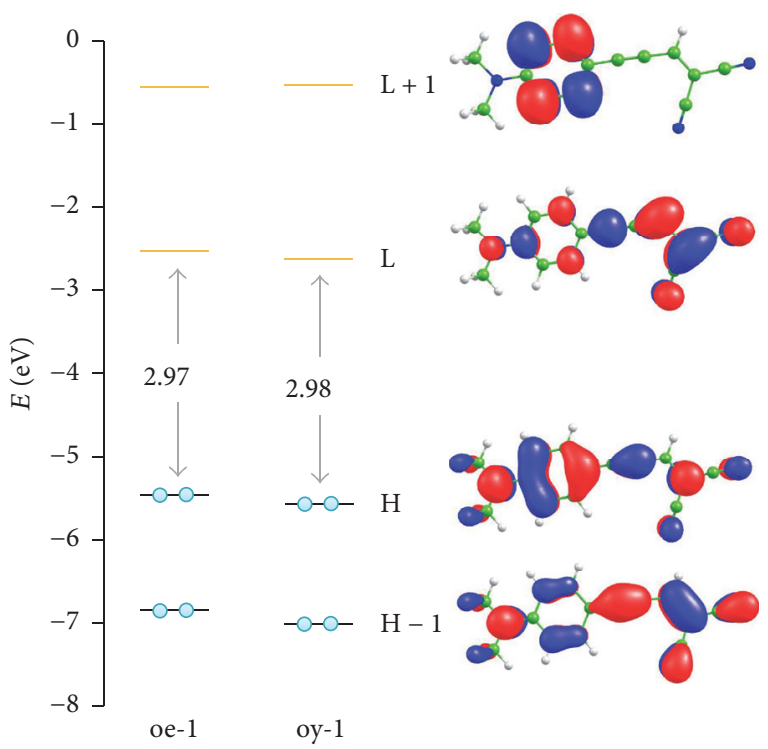

FIGURE 1: Frontier molecular orbital (MO) diagram of two representative compounds of the oligoene (oe- $n$ ) and oligoyne (oy- $n$ ) families. The topology of the frontier MOs is depicted in the case of oy- 1 . The $\mathrm{H}$ stands for HOMO whereas L stands for LUMO.

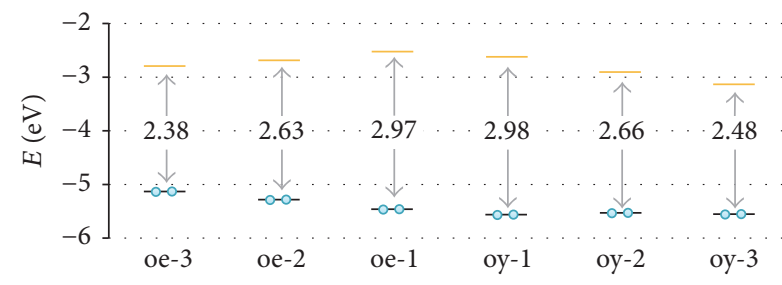

FIGURE 2: HOMO-LUMO gap evolution upon increasing the length and changing the type of the $\pi$-conjugated bridge.

is reduced in the oy- $n$ family going from oy- 1 to oy-3, for the latter being computed at $2.48 \mathrm{eV}$.

In order to analyse the effect of the geometry in the prediction of the lowest-lying CT electronic transition, two levels of theory were employed for obtaining the minimumenergy structure in the two families of compounds under study: the DFT-based B3LYP functional and the $a b$ initio MP2 method. The DFT methodology, especially when applied with hybrid functionals of the type of B3LYP, tends to provide structures unrealistically delocalized. It is well known that B3LYP underestimates the bond length alternation (BLA) of $\pi$-conjugated systems, showing large errors in the prediction of conjugation-dependent properties such as in vibrational or Raman spectroscopy [59-62]. In this regard, the MøllerPlesset MP2 approach usually offers high-quality BLA values, and stands as a reference for obtaining reliable geometries of polyconjugated systems [63]. Table 1 summarizes the main geometry parameters of the donor-acceptor compounds under study, that is, the bond length alternation and the pyramidalization of the $\mathrm{N}$ atom in the $\mathrm{N}, \mathrm{N}$-dimethylaniline group. 
TABLE 1: Characteristic geometry parameters of the fully optimized structures using B3LYP and MP2 compared to the lowest-lying CT electronic transition. See Scheme 2.

\begin{tabular}{|c|c|c|c|c|c|c|c|c|c|}
\hline & \multicolumn{4}{|c|}{ B3LYP geometry } & \multicolumn{4}{|c|}{ MP2 geometry } & \multirow{2}{*}{$S_{1, \exp }$} \\
\hline & $\mathrm{C}-\mathrm{N}-\mathrm{C}-\mathrm{C}^{\mathrm{a}}$ & $\mathrm{BLA}^{\mathrm{b}}$ & $S_{1}^{c}$ & $f$ & $\mathrm{C}-\mathrm{N}-\mathrm{C}-\mathrm{C}^{\mathrm{a}}$ & $\mathrm{BLA}^{\mathrm{b}}$ & $S_{1}^{c}$ & $f$ & \\
\hline oe-1 & 180 & 0.059 & 3.03 & 1.254 & 148.7 & 0.078 & 3.04 & 1.096 & $2.77^{\mathrm{d}}$ \\
\hline oe- 2 & 180 & 0.058 & 2.71 & 1.587 & 146.9 & 0.075 & 2.73 & 1.370 & $2.39^{\mathrm{d}}$ \\
\hline oe-3 & 180 & 0.057 & 2.46 & 1.880 & 145.5 & 0.073 & 2.50 & 1.636 & $2.30^{\mathrm{d}}$ \\
\hline oy-1 & 180 & 0.098 & 2.97 & 1.048 & 148.4 & 0.112 & 2.96 & 0.892 & $2.60^{\mathrm{e}}$ \\
\hline oy- 2 & 180 & 0.104 & 2.61 & 1.128 & 147.4 & 0.115 & 2.63 & 0.993 & $2.42^{\mathrm{d}}$ \\
\hline oy-3 & 180 & 0.105 & 2.34 & 1.077 & 147.0 & 0.116 & 2.31 & 0.944 & $2.30^{\mathrm{d}}$ \\
\hline
\end{tabular}

${ }^{\mathrm{a}}$ The pyramidalization of the amine group is measured by the dihedral angle C1-N1-C2-C3 (in degrees). ${ }^{\mathrm{b}}$ The bond length alternation (BLA, in $\AA$ ) is computed as described in Section 2. The calculation of the BLA for oe-1 is exemplified in the graph. ${ }^{\mathrm{c}}$ Theoretical $\left(S_{1}\right)$ and experimental $\left(S_{1, \exp }\right)$ energy of the lowest-lying CT electronic transition (in eV). ${ }^{\mathrm{d}}$ Data extracted from [12]. ${ }^{\mathrm{e}}$ Data from [23].

The B3LYP functional predicts in all structures that the amine group is perfectly planar as measured by the characteristic C-N-C-C dihedral angle of $180^{\circ}$ (see Table 1). However, the MP2 geometries indicate pyramidalization of the amide group to some extent. In fact, the C-N-C-C dihedral angle tends to decrease upon increasing the size of the $\pi$-conjugated bridge. In the oligoene family, this effect rapidly converges and, therefore, the reduction of the dihedral is computed of only $1^{\circ}$ moving from oe- 2 to oe-3. Otherwise, the increase in pyramidalization is computed to be almost negligible in the oligoyne family, with similar trends to those found for oligoenes (Table 1).

The BLA values computed for the B3LYP geometries were found to be smaller compared to those obtained from the MP2 geometries, as expected. In the oligoene family, the BLA values are computed in the $0.064-0.057 \AA$ range at the B3LYP geometries and are predicted to decrease upon increasing the bridge length. A similar trend is obtained for the MP2 geometries but with larger nominal values of BLA (range of $0.081-0.073 \AA$ ). An opposite trend is predicted for the oligoyne family. For example, the BLA value goes from $0.098 \AA$ in oy- 1 to $0.105 \AA$ in oy-3 at the B3LYP geometries. This trend, however, does not imply that the triple bonds strengthen and the single bonds weaken upon increasing the bridge length. A careful inspection of the bond distances showed that triple bonds lengthen whereas the single bonds of the bridge shorten as a consequence of the increased $\pi$ conjugation going from oy-1 to oy-3. By subtracting the last single/double bond (vinylene) grouping in the calculation of the BLA, the expected reduction is obtained: from 0.182 in oy-1 to 0.149 in oy- 2 and to $0.134 \AA$ in oy- 3 .

TD-DFT calculations were performed for the two families of compounds at the B3LYP/6-31G** level of theory in gas phase. Despite the noticeable differences predicted in the minimum-energy structure between the two approaches (B3LYP and MP2), practically negligible effect is found in the lowest-lying CT electronic transition energies (Table 1). Among the whole list of compounds, the largest difference between the B3LYP and MP2 geometries is found to be of only $0.04 \mathrm{eV}$ in the case of oe-3. Oscillator strengths are computed to be slightly lower in the case of the MP2 geometries. The theoretical excitation energies largely deviate from the experimentally reported CT band in the oligoene and oligoyne push-pull families, with an error of $\approx 0.4 \mathrm{eV}$ for the poorest performance obtained in oy-1 (Table 1). We hence conclude that there is a noticeable effect on the minimumenergy structure coming from optimizations using different approaches (DFT versus MP2). However, these differences do not impact the prediction of the absorption features for the push-pull chromophores under study. The adequate choice of the functional must be key for calculating predictive energies for the CT electronic band in these DMA- $\pi$-DCV chromophores.

3.2. DFT Performance. The performance of a set of functionals was therefore assessed in order to find an accurate yet affordable approach to tackle the prediction of the most important CT bands in push-pull chromophores of increasing size. The set of functionals was divided according to the following rungs: the pure-GGA functionals BLYP and PBE, the hybrid exchange-correlation functionals B3LYP and PBE0, the hybrid meta-GGA M06-2X, the range-separated HSE03, and the long-range corrected CAM-B3LYP and $w$ B97XD. The reference values for the CT electronic transition were obtained from the experimental data reported by Diederich and coworkers [12, 23].

The mean absolute deviation (MAD) calculated for the different functionals along the two families of compounds showed unexpectedly that the pure-GGA functionals performed the best with a MAD of $0.11 \mathrm{eV}$ for both BLYP and PBE. Otherwise, the MAD obtained for the hybrid analogues was computed to be 0.22 and $0.29 \mathrm{eV}$ for B3LYP and PBE0, respectively. The range-separated HSE03 behaved similarly, with a MAD value of $0.23 \mathrm{eV}$. Finally, the performance of the hybrid meta-GGA M06-2X and the long-range corrected CAM-B3LYP and wB97XD was found to be very poor, with MAD values of $0.51,0.54$, and $0.59 \mathrm{eV}$, respectively.

Due to this unexpected performance in the different rungs of density functionals, we analysed the effect of the solvation by using two different solvent models: the polarizable continuum model (PCM) and the solvent model based on solute density (SMD), using dichloromethane as a solvent. After the inclusion of the solvent effects, the MAD of the pure-GGA functionals turned out to increase up to $0.33 \mathrm{eV}$ for BLYP and $0.34 \mathrm{eV}$ for PBE. Interestingly, the hybrid functionals improved their performance with a MAD 


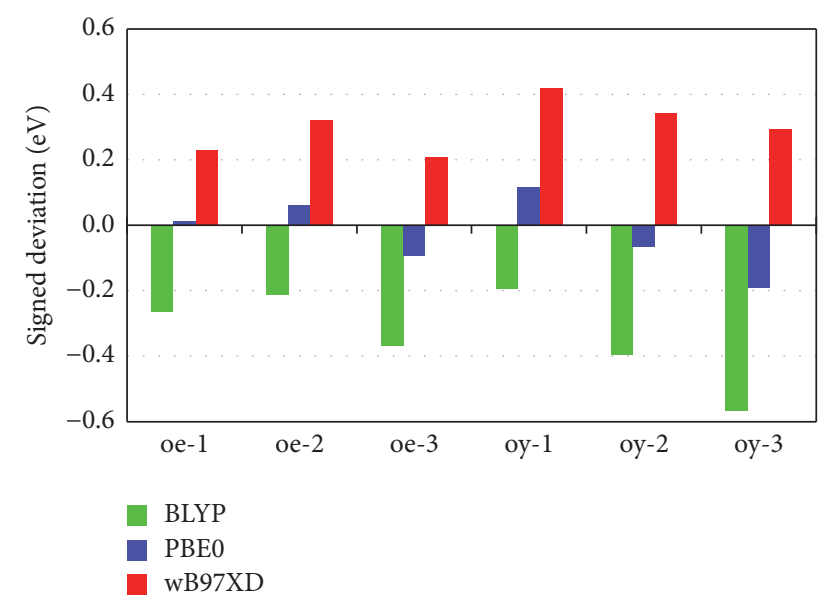

FIGURE 3: Signed deviation of representative pure-GGA, hybrid, and long-range corrected density functionals (using PCM and dichloromethane) in the prediction of the lowest-lying CT electronic excitation $S_{1}$ with respect to the experimental value.

of $0.11 \mathrm{eV}$ for B3LYP and the lowest MAD along the set of $0.08 \mathrm{eV}$ for PBE0. The HSE03, M06-2X, and the long-range corrected CAM-B3LYP and wB97XD also improved their performance compared to gas phase calculations, with MAD values of $0.12,0.20,0.23$, and $0.28 \mathrm{eV}$, respectively. Figure 3 summarizes the absolute deviations for representative functionals of the pure-GGA, hybrid, and long-range corrected rungs along the list of chromophores studied. In general, pure-GGA functionals underestimate the lowest-lying CT excitation, whereas the hybrid meta-GGA and the long-range corrected functionals overestimate it. This will be further analysed in terms of the percentage of the exact HartreeFock exchange below. The mean absolute deviations of the different rungs of functionals by using the SMD solvent model remained practically unchanged but with a small increase of the nominal error. Exceptions to this were the functionals M06-2X and CAM-B3LYP, whose performance improved with a MAD reduction of $0.03 \mathrm{eV}$. The effect of the solvent is therefore key for achieving a correct behaviour of the functional performance attending their rungs, although long-range corrected functionals should, in principle, predict the best CT energetics. This solvent effect is effectively captured by the two solvent models employed and will be consistently included by PCM hereafter.

The effect of the size and nature of the bridge was further analysed by comparing the different functionals of the set. In the oligoene family, an increase in the bridge size did not produce a trend in the performance of the list of functionals assessed (see Figure 3 for the case of BLYP, PBE0, and $\mathrm{wB} 97 \mathrm{XD})$. In this family, the signed deviations for the best performing PBE0 functional were computed to be $0.01,0.06$, and $-0.09 \mathrm{eV}$ going from oe- 1 to oe- 2 and to oe-3. Otherwise, for the oligoyne family, the PBE0 signed deviations were calculated from +0.12 to $-0.19 \mathrm{eV}$ along the oy- $n$ systems. The same behaviour was predicted in all the functionals. This trend suggests that, upon increasing the acetylene bridge, the DFT functionals underestimate progressively the CT

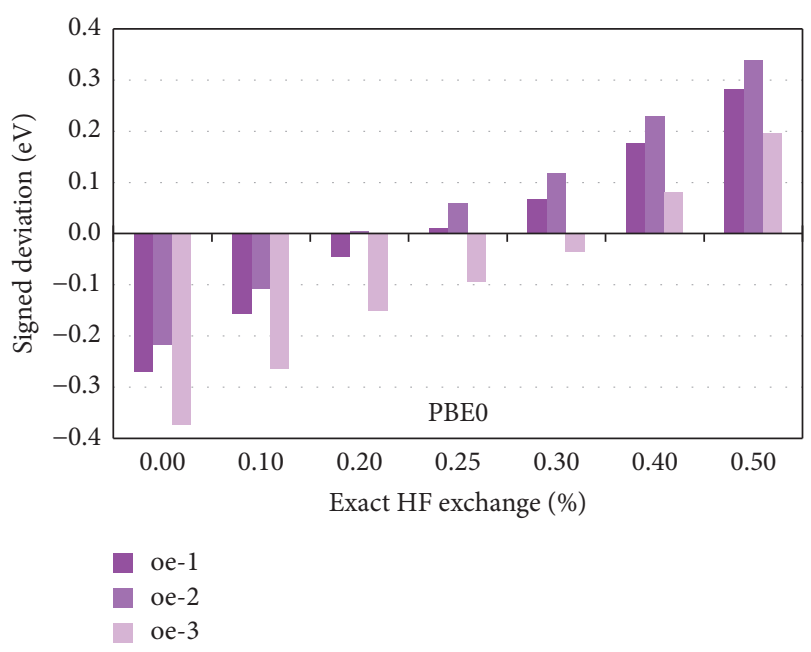

FIGURE 4: Signed deviation of the PBE family of functionals in the prediction of the lowest-lying CT state of oe- $n$ as a function of the $\%$ exact HF exchange.

excitation energy with respect to the experimental data. We postulate that the different type of $\pi$-conjugated bridge is important in the accurate prediction of the CT band by DFT. As widely recognised in the literature, CT excitations with low overlap between the orbitals participating in the transition are poorly described by conventional functionals [39]. This should in principle be solved by the long-range corrected functionals, which seems to be the case only for the oy- $n$ family: upon increasing the bridge length, a slight improvement in the performance of long-range corrected functionals is found (Figure 3).

We envisaged that the amount of the Hartree-Fock (\% $\mathrm{HF}$ ) exchange in the definition of the functional is key for the accurate prediction of the lowest-lying CT electronic transition. The expression for the widely used B3LYP and PBE0 functionals was employed to systematically increase the $\% \mathrm{HF}$ exchange going from 0 to $50 \%$ (see Section 2). TDDFT calculations were performed for both families of DMA$\pi$-DCV chromophores using variable \% of the HF exchange in the B3LYP and PBE0 expressions (see Figure 4 for the case of the PBE series in oe- $n$ ). The evolution found was that one would expect. For the PBE series, the mean deviation (MD) at $0 \% \mathrm{HF}$ (i.e., the PBE functional) is $-0.34 \mathrm{eV}$ (MAD $=0.34 \mathrm{eV}$ ), according to what was previously obtained for the pure-GGA PBE. Moving to higher values of $\% \mathrm{HF}$, the CT transition is predicted at larger energies and the MD reaches a value of $+0.30 \mathrm{eV}$ at $50 \% \mathrm{HF}(\mathrm{MAD}=0.30 \mathrm{eV})$. In between, we find that the best performance is obtained for 25 and $30 \%$ of the HF exchange with a MAD value of $0.09 \mathrm{eV}$ (see Figure 4). Interestingly, the amount of the \% HF exchange included in the original definition of the hybrid PBE0 functional (25\%) offers one of the best results in the prediction of the lowest-lying CT band for the DMA- $\pi$-DCV chromophores. The trends obtained for the PBE series are mirrored in the BLYP series. In this case, the BLYP with an amount of $30 \% \mathrm{HF}$ is found to provide the best MAD value of $0.09 \mathrm{eV}$ along the series. 
TABLE 2: Donor-acceptor distance $d$ (in $\AA$ ), $\Lambda$ diagnostic test, and PBE0 absolute error (in $\mathrm{eV}$ ) in the prediction of the lowest-lying CT electronic transition.

\begin{tabular}{lccc}
\hline Compound & $d^{\mathrm{a}}$ & $\Lambda$ index & PBE0 error \\
\hline oe-1 & 9.12 & 0.685 & 0.01 \\
oe-2 & 11.59 & 0.697 & 0.06 \\
oe-3 & 14.07 & 0.697 & -0.09 \\
oy-1 & 9.09 & 0.640 & 0.12 \\
oy-2 & 11.72 & 0.605 & -0.06 \\
oy-3 & 14.27 & 0.582 & -0.19 \\
\hline
\end{tabular}

${ }^{\mathrm{a}}$ Donor-acceptor distance measured as the distance between N1 and C4 (see Table 1 for numbering).

The effect of the orbital overlap in the CT electronic transition was examined by calculating the $\Lambda$ diagnostic test as detailed in Section 2. Table 2 summarizes the donor-acceptor distance and the $\Lambda$ index compared to the signed deviation of the best performing PBE0 functional upon increasing the bridge length. In the oligoene family, the orbital overlap between the HOMO and LUMO is unexpectedly preserved, or even increased, upon increasing the ethylene bridge length. This is evidenced by the $\Lambda$ index, which is computed in the $0.68-0.70$ range along oe- 1 to oe- 3 . Therefore, an increase in the ethylene bridge does not imply a decrease in the HOMOLUMO overlap, which is in line with the delocalized nature of the orbitals involved in these systems. The absolute value of the $\Lambda$ index for the oligoene family of compounds lies in the region where the hybrid functionals still perform well for CT excitation energies [39]. Although the distance between the donor and acceptor defined by the separation between $\mathrm{N} 1$ and C4 (see Table 1) significantly increases going from oe-1 to oe3 , the performance of the PBE0 functional is barely affected. The PBE0 signed deviation of PBE0 in oe-3 is computed to be $-0.09 \mathrm{eV}$, which does not correlate with the larger $\Lambda$ index compared to oe-1. Experimental deviations in the reference value for the CT transition in this compound may be the origin of this unexpected underestimation. Moving to the oligoyne compounds, the $\Lambda$ index is computed to reduce from 0.64 to 0.58 upon increasing the acetylene bridge from oy- 1 to oy- 3 . This is reflected by the PBE0 performance on the CT energetics, with a signed deviation that moves from +0.12 to -0.19 (Table 2). This trend is in line with the underestimation of the CT excitation energy by common functionals when the spatial overlap between the participating orbitals in the transition decreases. Still, however, the $\Lambda$ index for the CT electronic transition of the oligoyne family is also in an acceptable range for obtaining good predictions with conventional hybrid functionals; in fact, the maximum absolute deviation is computed to be lower than $0.20 \mathrm{eV}$ in the case of the largest bridge-containing oy-3. In comparison with the oligoene series, the $\mathrm{C} \equiv \mathrm{C}$ bridge in the oligoyne family separates the donor and acceptor moieties to a similar extent. This separation might be viewed as a reason for the underestimation of the CT band by PBE0 going from oy-1 to oy-3. However, the over/underestimation of the lowest-lying CT transition originates from the orbital overlap and, therefore, is reflected by the $\Lambda$ index.
TABLE 3: Solvatochromism in the two DMA- $\pi$-DCV families for five selected solvents. CT excitation energies (in $\mathrm{eV}$ ) are shown as calculated at the PBE0/6-31G ${ }^{* *}$ level of theory using the PCM solvent model.

\begin{tabular}{lcccccc}
\hline$\varepsilon$ & MCH & THF & DCM & MeOH & DMF & \multirow{2}{*}{$E_{\text {solv }}{ }^{\mathrm{a}}$} \\
\hline oe-1 & 2.0 & 7.4 & 8.9 & 32.6 & 37.2 & \\
oe-2 & 2.52 & 2.79 & 2.78 & 2.80 & 2.76 & 0.08 \\
oe-3 & 2.27 & 2.22 & 2.21 & 2.22 & 2.19 & 0.08 \\
oy-1 & 2.80 & 2.73 & 2.72 & 2.72 & 2.69 & 0.10 \\
oy-2 & 2.45 & 2.37 & 2.36 & 2.36 & 2.33 & 0.12 \\
oy-3 & 2.21 & 2.12 & 2.11 & 2.11 & 2.09 & 0.12 \\
\hline
\end{tabular}

${ }^{\mathrm{a}}$ The largest CT excitation energy difference is calculated between solvents $\mathrm{MCH}$ and DMF to measure the amount of solvatochromism between the different chromophores as a function of the bridge length and nature.

Optical linear and nonlinear properties of common push-pull molecules mostly depend on the polarizability of the electrons localized in $\pi$-bonding molecular orbitals. Although the polarizability of a molecule is mainly given by its chemical structure, in particular by the length of the $\pi$ conjugated spacer and the electronic nature of the donors and acceptors attached, it can also be affected by external factors such as the strength of radiation and state of matter and in solution also by the solvent used. We have shown above that, by including the solvent effect through a continuum model, accurate CT excitation energies for the widely used hybrid functionals are obtained. The solvatochromism of the most relevant low-lying $\mathrm{CT}$ electronic transition in the oligoene and oligoyne families of compounds is investigated using the best performing PBE0 functional by means of the PCM solvent model with the following solvents: methylcyclohexane $(\mathrm{MCH}, \varepsilon=2.0$ ), tetrahydrofuran (THF, $\varepsilon=7.4$ ), dichloromethane (DCM, $\varepsilon=8.9)$, methanol $(\mathrm{MeOH}, \varepsilon=$ 32.6), and $\mathrm{N}, \mathrm{N}$-dimethylformamide (DMF, $\varepsilon=37.2$ ).

Table 3 summarizes the excitation energies calculated for the lowest-lying CT transition along the oligoene and oligoyne chromophores. In the oligoene family, the CT electronic transition moves to lower energies upon going from solvents with low to high dielectric constant (Table 3). The largest difference $\left(\Delta E_{\text {solv }}\right)$ is predicted between the $\mathrm{MCH}$ and DMF solvents, which gives a red shift of $0.08 \mathrm{eV}$ for oe$n$. The same behaviour is found in the oligoyne series, but now the solvatochromism is predicted to be larger, with a $\Delta E_{\text {solv }}$ value that increases from 0.10 in oy- 1 to 0.12 oy- 3 upon increasing the bridge size. Interestingly, the evolution of the $\Delta E_{\text {solv }}$ matches the trends described for the $\Lambda$ diagnostic test, which is a direct measure of the HOMO-LUMO spatial overlap. Whereas in the oligoene family both HOMO and LUMO largely overlap, the lower $\pi$-conjugation of the acetylene bridge in the oligoyne family disfavours this overlap upon increasing the bridge size and, as a consequence, the solvatochromism is found to be more pronounced. Figure 5 displays the solvatochromism calculated for the oy-3 chromophore suggesting that the change in solvent should not impact significantly the intensity of the most relevant CT band. Discrete solvent-solute interactions might however 


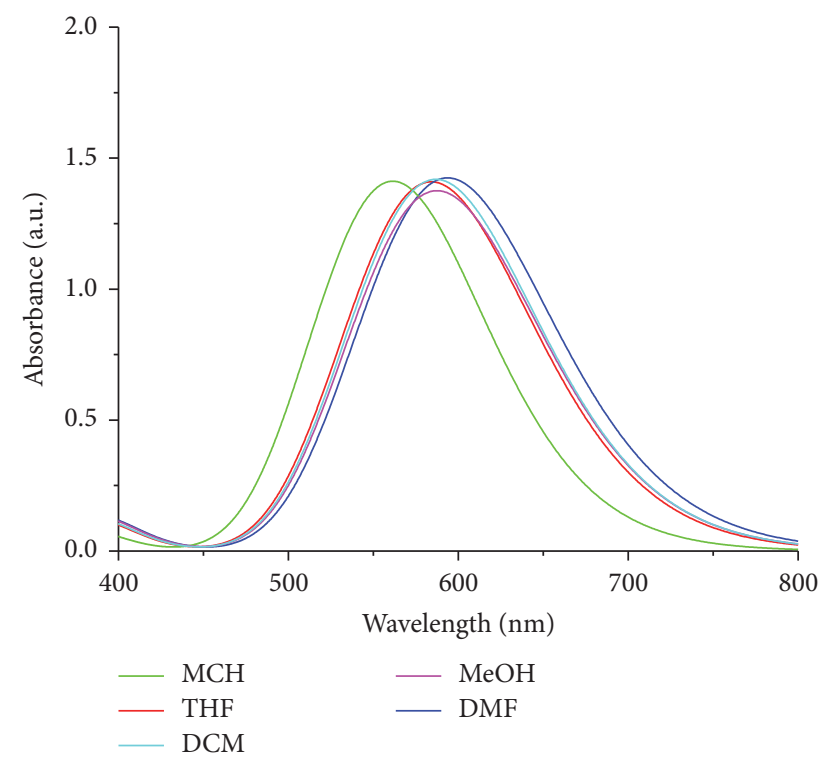

FIGURE 5: Convoluted absorption spectra in the region of the lowestlying CT electronic transition calculated at the PBE0/6-31G ${ }^{* *}$ level using PCM and a variety of solvents for the oy-3 chromophore. A full width at half maximum of $0.20 \mathrm{eV}$ was used in the Gaussian functions for the convolution.

lead to slight intensity dependence, which is out of the scope of the present study.

3.3. The Case of the TTF-TCB-NMe 2 . Despite the great success and appealing properties of planar push-pull chromophores such as the oligoene oe- $n$ and oligoyne oy- $n$ families, great effort has been devoted to the synthesis of nonplanar charge-transfer chromophores that exhibit desirable physical properties compared to their planar counterparts: they are usually more soluble, less aggregating, and more readily sublimable, forming amorphous rather than crystalline films for potential use in optoelectronic devices. A TTF-based derivative was recently synthesized containing a tetracyanobutadiene moiety (TTF-TCB-H, Scheme 1). This compound was demonstrated to exhibit a moderately intense, broad charge-transfer band in the 700-900 $\mathrm{nm}$ range originated by one-electron promotion from the TTF to the TCB unit. The substitution of the $\mathrm{H}$ in para position of the benzene ring by $\mathrm{N}, \mathrm{N}$-dimethylaniline (DMA) group led to a compound (TTF-TCB-NMe $\mathrm{N}_{2}$, Scheme 1) preserving the TTF $\rightarrow$ TCB CT band together with the emergence of a new highly intense, sharp CT transition in the region of $450-550 \mathrm{~nm}$. This system constitutes a great example to test the performance of a set of density functionals for the prediction of its two low-lying CT bands located separately, with different relative intensities and nature.

The minimum-energy structure of TTF-TCB-H and TTF-TCB-NMe ${ }_{2}$ was calculated at the B3LYP/6-31G ${ }^{* *}$ level of theory in gas phase. These two chromophores show spatial disposition highly distorted from planarity due to the steric hindrance promoted by the tetracyanobutadiene linker. In fact, the two donor moieties (DMA and TTF) are practically perpendicular through the $\pi$-conjugated path (see
Figure 6(a) for the case of TTF-TCB-NMe ${ }_{2}$ ). This conformation leads to partial breaking of the $\pi$-conjugation and provokes highly localized molecular orbitals. The reliability of the theoretical minimum-energy geometries was confirmed by the good accord of the TTF-TCB-H structure with the available X-ray crystallographic data [11].

The electronic structure of the two push-pull systems was analysed on the minimum-energy geometries previously optimized. Theoretical calculations on the TTF-TCB-NMe indicate that the highest-occupied molecular orbitals HOMO and HOMO - 1 are mostly localized on the donor TTF and DMA moieties, respectively, with a minor contribution of the TCB acceptor bridge. Otherwise, the lowest-unoccupied molecular orbitals LUMO and LUMO +1 are highly localized on the TCB fragment, spreading slightly to the TTF moiety in the case of LUMO and to the DMA in the case of LUMO + 1 (Figure 6(b)). For TTF-TCB-H, both HOMO and HOMO -1 are localized on the TTF moiety, whereas LUMO and LUMO + 1 are in the TCB unit. TDDFT calculations were preliminarily performed at the $\mathrm{B} 3 \mathrm{LYP} / 6-31 \mathrm{G}^{* *}$ level to identify the most characteristic transitions that lead to the optical features of TTF-TCB-NMe $\mathrm{N}_{2}$. The one-electron promotion from the HOMO to the LUMO is responsible for the singlet $S_{1}$ CT state, which is experimentally recorded at $c a .700 \mathrm{~nm}$ $(1.77 \mathrm{eV})[11]$, and reasonably agrees with the HOMO-LUMO gap calculated at $2.05 \mathrm{eV}$. The intense $\mathrm{CT}$ band experimentally recorded at $450 \mathrm{~nm}(2.76 \mathrm{eV})$ corresponds however to the electron promotion from the HOMO -1 to the LUMO + 1 , in which the energy difference is calculated to be $3.06 \mathrm{eV}$ at the B3LYP/6-31G ${ }^{* *}$ level. This electronic excitation belongs to the singlet $S_{5}$ excited state and is computed with an oscillator strength $(f)$ of 0.744 . Energetically in between are calculated states $S_{2}, S_{3}$, and $S_{4}$, which correspond to weakly intense $(f<$ $0.012)$ CT $\left(S_{2}\right.$ and $\left.S_{3}\right)$ and TTF-centred $\left(S_{4}\right)$ transitions. For TTF-TCB-H, no intense electronic excitations are predicted in the region of $500 \mathrm{~nm}$, in good accord with the experimental evidence [11].

The spatial overlap of the two characteristic chargetransfer electronic transitions in TTF-TCB-NMe ${ }_{2}$ was calculated to anticipate the performance of conventional density functionals in their prediction. The $\Lambda$ index of the TTF $\rightarrow$ TCB transition $\left(S_{1}\right)$ is calculated at 0.42 in TTF-TCB-H and 0.46 in TTF-TCB-NMe ${ }_{2}$. Despite being very similar, theoretical calculations indicate that the inclusion of the DMA group slightly favours the overlap between the two orbitals (HOMO and LUMO) participating in the lowest-lying CT excitation. The $\Lambda$ index for the low-lying DMA $\rightarrow$ TCB CT transition $\left(S_{5}\right)$ is computed to be higher, at 0.62 for TTF-TCB-NMe ${ }_{2}$, suggesting a moderate spatial overlap between the DMA and TCB-centred molecular orbitals (Figure 6(b)). $\Lambda$ values for both $S_{1}$ and $S_{5}$ electronic transitions suggest that hybrid functionals might provide a reasonably good performance for these CT states.

In order to analyse the performance of different density functionals in the prediction of the two characteristic CT electronic transitions of TTF-TCB-NMe ${ }_{2}$, we selected the $\mathrm{BLYP}, \mathrm{PBE0}$, and $\mathrm{wB} 97 \mathrm{XD}$ as representative functionals of pure-GGA, hybrid, and long-range corrected rungs. Solvent effects were included within the PCM model and using 


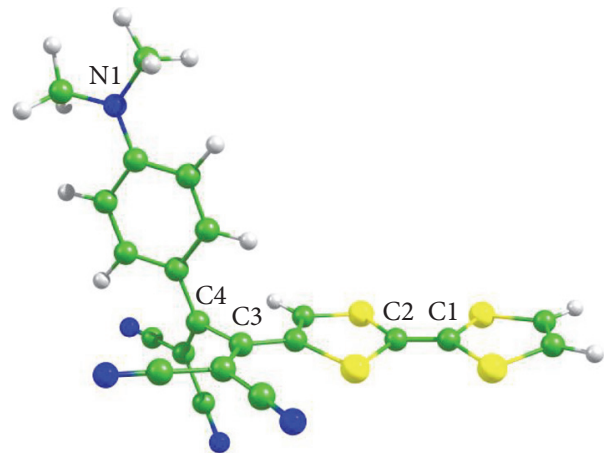

(a)
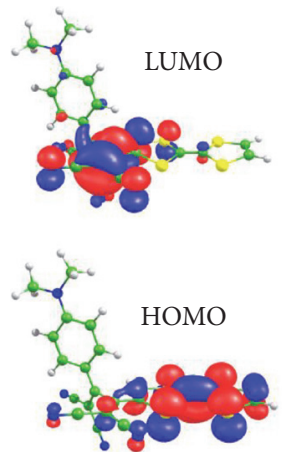

(b)

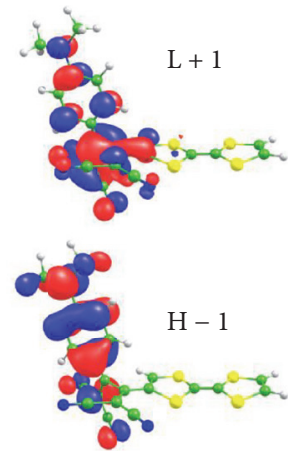

Figure 6: (a) Minimum-energy geometry of TTF-TCB-NMe ${ }_{2}$ calculated at the B3LYP/6-31G ${ }^{* *}$ level of theory in gas phase. Colouring: $\mathrm{S}$ atoms in yellow, $\mathrm{C}$ atoms in green, and $\mathrm{N}$ atoms in blue. (b) Frontier molecular orbital topologies calculated at the same level using an isovalue of 0.03 .

TABLE 4: Donor-acceptor distance $d$ (in $\AA$ ), $\Lambda$ index, and signed deviations (in $\mathrm{eV}$ ) of three representative density functionals compared to the absolute experimental values of the low-lying CT electronic transitions $S_{1}$ (TTF $\rightarrow$ TCB) and $S_{5}$ (DMA $\rightarrow$ TCB).

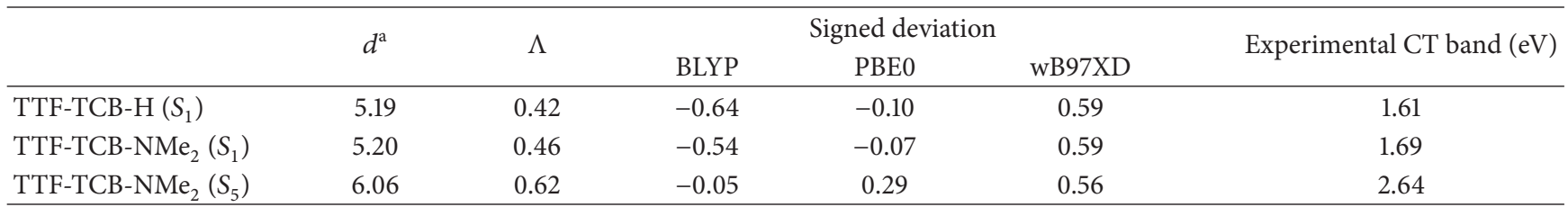

${ }^{\mathrm{a}}$ The donor-acceptor distance (in $\AA$ ) is measured as the distance between the C1-C2 centroid and the C3-C4 centroid for $S_{1}$ and between the N1 and the C3-C4 centroid for $S_{5}$ (see Figure 6(a) for numbering).

dichloromethane. Table 4 summarizes the signed errors of the three functionals on the two intense CT transitions compared with the experimental data.

The lowest-lying CT electronic transition, recorded at $1.61 \mathrm{eV}$ experimentally, is well predicted by hybrid functionals as PBE0 in both compounds TTF-TCB-H and TTFTCB- $\mathrm{NMe}_{2}$, with absolute deviations as small as -0.10 and $-0.07 \mathrm{eV}$, respectively. Similar performance was confirmed by the B3LYP functional, with errors of -0.20 and $-0.16 \mathrm{eV}$. Otherwise, pure-GGA BLYP functional behaves poorly, with large deviations of -0.64 and $-0.54 \mathrm{eV}$ for TTF-TCB-H and TTF-TCB-NMe ${ }_{2}$, respectively, compared with the reference data (Table 4). Surprisingly, and in line with what is already found in oligoene and oligoyne derivatives, the long-range corrected wB97XD performs as bad as BLYP, in this case with positive deviations of $0.59 \mathrm{eV}$. The spatial overlap between the orbitals participating in $S_{1}$ (HOMO $\rightarrow$ LUMO) is therefore large enough for using hybrid functionals, which in fact outperform other types of density functional rungs.

Moving to the low-lying CT transition $S_{5}$ originated by the donor DMA $\rightarrow$ acceptor TCB electron promotion, the hybrid PBE0 functional provides an absolute deviation of $0.29 \mathrm{eV}$. The long-range corrected wB97XD performs again poorly, with a deviation of $0.56 \mathrm{eV}$ compared with the experimental reference (Table 4). For this electronic transition, the pure-GGA BLYP outperforms the rest of the rungs, with a signed deviation of only $-0.05 \mathrm{eV}$. The $\Lambda$ diagnostic test for this excitation was computed to be as large as 0.62 and, therefore, falls into the range for which pure-GGA might provide accurate electronic excitations of
CT nature. However, as already predicted in the linear DMA$\pi$-DCV chromophores, pure-GGA functionals performed with absolute deviations larger than $0.2 \mathrm{eV}$ for the donor $\rightarrow$ acceptor, DMA $\rightarrow$ DCV transition, with $\Lambda$ indexes ranging from 0.58 to 0.70 (Figure 3 and Table 2).

Theoretical calculations were carried out for the TTFTCB-NMe ${ }_{2}$ system by removing the donor TTF unit (DMATCB) [8]. This compound was reoptimized, and TDDFT calculations were performed at the same level of theory. Signed deviations on the lowest-lying CT electronic transition $S_{1}$ were computed for the BLYP and PBE0 functionals. Whereas the hybrid PBE0 functional performs with an error of $-0.42 \mathrm{eV}$, similarly as previously obtained for the DMA $\rightarrow$ TCB transition in TTF-TCB-NMe ${ }_{2}$, the pure-GGA predicts an excitation energy that largely deviates from the reference (error of $-0.58 \mathrm{eV}$ ). In fact, a $\Lambda$ index of 0.50 is calculated for this transition, which rules out a possible low overlap between the orbitals involved in the excitation. These results point to an error compensation in the case of pure-GGA functionals that lead to an abnormal small error in the prediction of the low-lying CT DMA $\rightarrow$ TCB band for TTF-TCB-NMe 2 .

\section{Conclusions}

Herein, we report on the optical features of a list of pushpull chromophores based on the donor $\mathrm{N}, \mathrm{N}$-dimethylaniline and acceptor dicyanovinylene moieties through calculations using density functional theory. Focus has been made on the performance of different rung of density functionals in predicting the lowest-lying charge-transfer electronic excitation. 
The comparison of two families of linear chromophores, oligoenes versus oligoynes, allowed disentangling the effect of the type and size of the $\pi$-conjugated bridge that separates the donor and acceptor fragments. An increase of the ethylene bridge in oligoenes was shown to infer a reduction of the HOMO-LUMO gap, with a direct consequence on the energetics of the lowest-lying CT electronic transition. Similarly, increasing the acetylene bridge in oligoynes led to a decrease in the gap, but only as a consequence of the stabilization of the LUMO orbital. The $\Lambda$ diagnostic test revealed that oligoenes provide a large overlap between the orbitals participating in the CT transition, and this overlap is maintained upon increasing the bridge length. Contrarily, an increase in the acetylene bridge length leads to systematic lowering of the donor-acceptor orbital spatial overlap due to its poorer $\pi$ communication compared with the ethylene bridge. Among the different rungs of density functionals assessed, the hybrid exchange-correlation functionals (B3LYP and PBE0) showed the most promising results in the prediction of the low-lying CT bands, with absolute deviations of $<0.20 \mathrm{eV}$. Surprisingly, long-range corrected functionals provided large errors of $>0.20 \mathrm{eV}$ in all cases. The percentage of exact Hartree-Fock exchange in the definition of the functional was demonstrated to dramatically affect the CT excitation energy, which showed a very good balance in the case of the best performing PBE0. The solvatochromism in the oligoene and oligoyne pushpull systems was confirmed by making use of a continuum solvent model, with a red shift of the characteristic CT band upon increasing the dielectric constant of the solvent. This effect was predicted to be larger in the family of the oligoynes chromophores presumably due to the larger localization of the HOMO and LUMO in the donor and acceptor moieties, respectively. The donor-acceptor-donor nonplanar TTF-TCB-NMe ${ }_{2}$ chromophore was finally used as a test compound, providing two CT bands of different nature and situated at different range of energy in the absorption spectrum. Again, hybrid density functionals showed the best consistent performance in the prediction of both the TTF $\rightarrow$ TCB and DMA $\rightarrow$ TCB charge-transfer bands. Long-range corrected functionals systematically led to large positive deviations for the CT energetics and are therefore not recommended for use in this (and related) family of compounds.

\section{Competing Interests}

The author declares that there are no competing interests regarding the publication of this paper.

\section{Acknowledgments}

This work is supported by the Ministerio de Economía y Competitividad (MINECO) of Spain (Project CTQ201452045-R) and Unidad María de Maeztu (MDM-2015-0538). The author acknowledges the Spanish Ministry of Education, Culture, and Sport (MECD) for an FPU grant and thanks the QCMATMOL group headed by Professor E. Ortí for support and computer resources.

\section{References}

[1] J. M. Hales, S. Barlow, H. Kim et al., "Design of organic chromophores for all-optical signal processing applications," Chemistry of Materials, vol. 26, no. 1, pp. 549-560, 2014.

[2] M. Murai, S.-Y. Ku, N. D. Treat, M. J. Robb, M. L. Chabinyc, and C. J. Hawker, "Modulating structure and properties in organic chromophores: influence of azulene as a building block," Chemical Science, vol. 5, no. 10, pp. 3753-3760, 2014.

[3] S. R. Marder, B. Kippelen, A. K.-Y. Jen, and N. Peyghambarian, "Design and synthesis of chromophores and polymers for electro-optic and photorefractive applications," Nature, vol. 388, no. 6645, pp. 845-851, 1997.

[4] L. Levi and T. J. J. Müller, "Multicomponent syntheses of functional chromophores," Chemical Society Reviews, vol. 45, no. 10, pp. 2825-2846, 2016.

[5] S. Barlow and S. R. Marder, "Nonlinear optical properties of organic materials," in Functional Organic Materials: Syntheses, Strategies and Applications, T. J. J. Müller and U. H. F. Bunz, Eds., chapter 11, pp. 393-437, Wiley-VCH, Weinheim, Germany, 2006.

[6] R. R. Tykwinski, U. Gubler, R. E. Martin, F. Diederich, C. Bosshard, and P. Günter, "Structure-property relationships in third-order nonlinear optical chromophores," The Journal of Physical Chemistry B, vol. 102, no. 23, pp. 4451-4465, 1998.

[7] U. Gubler, R. Spreiter, C. Bosshard, P. Günter, R. R. Tykwinski, and F. Diederich, "Two-dimensionally conjugated molecules: the importance of low molecular symmetry for large thirdorder nonlinear optical effects," Applied Physics Letters, vol. 73, no. 17, pp. 2396-2398, 1998.

[8] T. Michinobu, C. Boudon, J.-P. Gisselbrecht et al., "Donorsubstituted 1,1,4,4-tetracyanobutadienes (TCBDs): new chromophores with efficient intramolecular charge-transfer interactions by atom-economic synthesis," Chemistry - A European Journal, vol. 12, no. 7, pp. 1889-1905, 2006.

[9] N. N. P. Moonen, W. C. Pomerantz, R. Gist et al., "Donorsubstituted cyanoethynylethenes: $\pi$-conjugation and band-gap tuning in strong charge-transfer chromophores," Chemistry-A European Journal, vol. 11, no. 11, pp. 3325-3341, 2005.

[10] H. Meier, J. Gerold, H. Kolshorn, and B. Mühling, "Extension of conjugation leading to bathochromic or hypsochromic effects in OPV series," Chemistry-A European Journal, vol. 10, no. 2, pp. 360-370, 2004.

[11] S.-I. Kato, M. Kivala, W. B. Schweizer, C. Boudon, J.-P. Gisselbrecht, and F. Diederich, "Origin of intense intramolecular charge-transfer interactions in nonplanar push-pull chromophores," Chemistry - A European Journal, vol. 15, no. 35, pp. 8687-8691, 2009.

[12] B. B. Frank, P. R. Laporta, B. Breiten et al., "Comparison of CC triple and double bonds as spacers in push-pull chromophores," European Journal of Organic Chemistry, no. 23, pp. 4307-4317, 2011.

[13] R. Gómez, C. Seoane, and J. L. Segura, "The first two decades of a versatile electron acceptor building block: 11,11,12,12tetracyano-9,10-anthraquinodimethane (TCAQ)," Chemical Society Reviews, vol. 36, no. 8, pp. 1305-1322, 2007.

[14] A. Ortíz, B. Insuasty, M. R. Torres et al., "Aminopyrimidinebased donor-acceptor chromophores: push-pull versus aromatic behaviour," European Journal of Organic Chemistry, vol. 2008, no. 1, pp. 99-108, 2008. 
[15] B. Wang, Y. Wang, J. Hua et al., "Starburst triarylamine donoracceptor-donor quadrupolar derivatives based on cyanosubstituted diphenylaminestyrylbenzene: tunable aggregationinduced emission colors and large two-photon absorption cross sections," Chemistry - A European Journal, vol. 17, no. 9, pp. 2647-2655, 2011.

[16] S. J. Cha, N. S. Han, J. K. Song, S.-R. Park, Y. M. Jeon, and M. C. Suh, "Efficient deep blue fluorescent emitter showing high external quantum efficiency," Dyes and Pigments, vol. 120, pp. 200-207, 2015.

[17] A. Pron, P. Gawrys, M. Zagorska, D. Djurado, and R. Demadrille, "Electroactive materials for organic electronics: preparation strategies, structural aspects and characterization techniques," Chemical Society Reviews, vol. 39, no. 7, pp. 2577$2632,2010$.

[18] S.-L. Suraru and F. Würthner, "Strategies for the synthesis of functional naphthalene diimides," Angewandte ChemieInternational Edition, vol. 53, no. 29, pp. 7428-7448, 2014.

[19] A. Szłapa, S. Kula, U. Błaszkiewicz, M. Grucela, E. SchabBalcerzak, and M. Filapek, "Simple donor- $\pi$-acceptor derivatives exhibiting aggregation-induced emission characteristics for use as emitting layer in OLED," Dyes and Pigments, vol. 129, pp. 80-89, 2016.

[20] N. N. P. Moonen, C. Boudon, J.-P. Gisselbrecht, P. Seiler, M. Gross, and F. Diederich, "Cyanoethynylethenes: a class of powerful electron acceptors for molecular scaffolding," Angewandte Chemie-International Edition, vol. 41, no. 16, pp. 3044-3047, 2002.

[21] N. N. P. Moonen, R. Gist, C. Boudon et al., "Donorsubstituted cyanoethynylethenes: powerful chromophores for opto-electronic applications," Organic and Biomolecular Chemistry, vol. 1, no. 12, pp. 2032-2034, 2003.

[22] M. Kivala, C. Boudon, J.-P. Gisselbrecht, P. Seiler, M. Gross, and F. Diederich, "A novel reaction of 7,7,8,8-tetracyanoquinodimethane (TCNQ): charge-transfer chromophores by [2+2] cycloaddition with alkynes," Chemical Communications, no. 45 , pp. 4731-4733, 2007.

[23] T. Michinobu, J. C. May, J. H. Lim et al., "A new class of organic donor-acceptor molecules with large third-order optical nonlinearities," Chemical Communications, no. 6, pp. 737-739, 2005.

[24] M. Kivala, T. Stanoeva, T. Michinobu, B. Frank, G. Gescheidt, and F. Diederich, "One-electron-reduced and -oxidized stages of donor-substituted 1,1,4,4-tetracyanobuta-1,3-dienes of different molecular architectures," Chemistry - A European Journal, vol. 14, no. 25, pp. 7638-7647, 2008.

[25] T. Gibtner, F. Hampel, J.-P. Gisselbrecht, and A. Hirsch, "Endcap stabilized oligoynes: model compounds for the linear sp carbon allotrope carbyne," Chemistry, vol. 8, no. 2, pp. 408-432, 2002.

[26] J.-P. Gisselbrecht, N. N. P. Moonen, C. Boudon, M. B. Nielsen, F. Diederich, and M. Gross, "Redox properties of linear and cyclic scaffolds based on di- and tetraethynylethene," European Journal of Organic Chemistry, vol. 2004, no. 14, pp. 2959-2972, 2004.

[27] K. Qvortrup, M. T. Jakobsen, J.-P. Gisselbrecht et al., "Donor strength of $\pi$-extended tetrathiafulvalenes: ionisation energies vs. oxidation potentials. A joint theoretical and experimental study," Journal of Materials Chemistry, vol. 14, no. 11, pp. 17681773, 2004.

[28] J. Calbo, P. M. Viruela, and E. Ortí, “Theoretical insight on novel donor-acceptor exTTF-based dyes for dye-sensitized solar cells," Journal of Molecular Modeling, vol. 20, no. 4, article 2188, 2014.

[29] R. García, M. Á. Herranz, M. R. Torres et al., “Tuning the electronic properties of nonplanar exTTF-based pusha-pull chromophores by aryl substitution," The Journal of Organic Chemistry, vol. 77, no. 23, pp. 10707-10717, 2012.

[30] P.-A. Bouit, L. Infantes, J. Calbo et al., "Efficient light harvesters based on the 10-(1,3-dithiol-2-ylidene)anthracene core," Organic Letters, vol. 15, no. 16, pp. 4166-4169, 2013.

[31] J. Calbo, J. Aragó, and E. Ortí, “Theoretical study of the benzoquinone-tetrathiafulvalene- benzoquinone triad in neutral and oxidized/reduced states," Theoretical Chemistry Accounts, vol. 132, no. 3, pp. 1-10, 2013.

[32] J. Calbo, J. Aragó, F. Otón et al., “Tetrathiafulvalene-based mixed-valence acceptor-donor-acceptor triads: a joint theoretical and experimental approach," Chemistry-A European Journal, vol. 19, pp. 16656-16664, 2013.

[33] J. Calbo, M. Pastore, E. Mosconi, E. Ortí, and F. De Angelis, "Computational modeling of single- versus double-anchoring modes in di-branched organic sensitizers on $\mathrm{TiO}_{2}$ surfaces: structural and electronic properties," Physical Chemistry Chemical Physics, vol. 16, no. 10, pp. 4709-4719, 2014.

[34] Computer Aided Innovation of New Materials, Elsevier, Oxford, $\mathrm{UK}, 1991$.

[35] J. Preat, D. Jacquemin, D. P. Vercauteren, and E. A. Perpète, "A quantitative prediction of the electronic spectra of thiocarbonyl chromophores: TD-DFT versus SAC-CI," Theoretical Chemistry Accounts, vol. 119, no. 5-6, pp. 463-468, 2008.

[36] D. J. Tozer, R. D. Amos, N. C. Handy, B. O. Roos, and L. Serrano-Andrés, "Does density functional theory contribute to the understanding of excited states of unsaturated organic compounds?” Molecular Physics, vol. 97, no. 7, pp. 859-868, 1999.

[37] A. Dreuw and M. Head-Gordon, "Failure of time-dependent density functional theory for long-range charge-transfer excited states: the zincbacteriochlorin-bacteriochlorin and bacteriochlorophyll-spheroidene complexes," Journal of the American Chemical Society, vol. 126, no. 12, pp. 4007-4016, 2004.

[38] T. Tsuneda and K. Hirao, "Long-range correction for density functional theory," Wiley Interdisciplinary Reviews: Computational Molecular Science, vol. 4, no. 4, pp. 375-390, 2014.

[39] M. J. G. Peach, P. Benfield, T. Helgaker, and D. J. Tozer, "Excitation energies in density functional theory: an evaluation and a diagnostic test," The Journal of Chemical Physics, vol. 128, no. 4, Article ID 044118, 2008.

[40] A. D. Becke, "Density-functional thermochemistry. III. The role of exact exchange," The Journal of Chemical Physics, vol. 98, no. 7, pp. 5648-5652, 1993.

[41] M. M. Francl, W. J. Pietro, W. J. Hehre et al., "Self-consistent molecular orbital methods. XXIII. A polarization-type basis set for second-row elements," The Journal of Chemical Physics, vol. 77, no. 7, pp. 3654-3665, 1982.

[42] C. Jamorski, M. E. Casida, and D. R. Salahub, "Dynamic polarizabilities and excitation spectra from a molecular implementation of time-dependent density-functional response theory: $\mathrm{N}_{2}$ as a case study," The Journal of Chemical Physics, vol. 104, no. 13, pp. 5134-5147, 1996.

[43] M. E. Casida, C. Jamorski, K. C. Casida, and D. R. Salahub, "Molecular excitation energies to high-lying bound states from 
time-dependent density-functional response theory: characterization and correction of the time-dependent local density approximation ionization threshold," Journal of Chemical Physics, vol. 108, no. 11, pp. 4439-4449, 1998.

[44] M. Petersilka, U. J. Gossmann, and E. K. U. Gross, "Excitation energies from time-dependent density-functional theory," Physical Review Letters, vol. 76, no. 8, pp. 1212-1215, 1996.

[45] M. Head-Gordon, J. A. Pople, and M. J. Frisch, "MP2 energy evaluation by direct methods," Chemical Physics Letters, vol. 153, no. 6, pp. 503-506, 1988.

[46] J. P. Perdew, K. Burke, and M. Ernzerhof, "Generalized gradient approximation made simple," Physical Review Letters, vol. 77, no. 18, pp. 3865-3868, 1996.

[47] A. D. Becke, "Density-functional exchange-energy approximation with correct asymptotic behavior," Physical Review A, vol. 38, no. 6, pp. 3098-3100, 1988.

[48] C. Lee, W. Yang, and R. G. Parr, "Development of the ColleSalvetti correlation-energy formula into a functional of the electron density," Physical Review B, vol. 37, no. 2, pp. 785-789, 1988.

[49] C. Adamo and V. Barone, "Toward reliable density functional methods without adjustable parameters: the PBE0 model," The Journal of Chemical Physics, vol. 110, no. 13, pp. 6158-6170, 1999.

[50] Y. Zhao and D. G. Truhlar, "The M06 suite of density functionals for main group thermochemistry, thermochemical kinetics, noncovalent interactions, excited states, and transition elements: two new functionals and systematic testing of four M06-class functionals and 12 other functionals," Theoretical Chemistry Accounts, vol. 120, no. 1-3, pp. 215-241, 2008.

[51] J. Heyd, G. E. Scuseria, and M. Ernzerhof, "Hybrid functionals based on a screened Coulomb potential," The Journal of Chemical Physics, vol. 118, no. 18, pp. 8207-8215, 2003.

[52] T. Yanai, D. P. Tew, and N. C. Handy, "A new hybrid exchangecorrelation functional using the Coulomb-attenuating method (CAM-B3LYP)," Chemical Physics Letters, vol. 393, no. 1-3, pp. 51-57, 2004.

[53] J.-D. Chai and M. Head-Gordon, "Long-range corrected hybrid density functionals with damped atom-atom dispersion corrections," Physical Chemistry Chemical Physics, vol. 10, no. 44, pp. 6615-6620, 2008.

[54] R. Ditchfield, W. J. Hehre, and J. A. Pople, "Self-consistent molecular-orbital methods. IX. An extended gaussian-type basis for molecular-orbital studies of organic molecules," The Journal of Chemical Physics, vol. 54, no. 2, pp. 720-723, 1971.

[55] A. D. McLean and G. S. Chandler, "Contracted Gaussian basis sets for molecular calculations. I. Second row atoms, $Z=11-18$," The Journal of Chemical Physics, vol. 72, pp. 5639-5648, 1980.

[56] G. Scalmani and M. J. Frisch, "Continuous surface charge polarizable continuum models of solvation. I. General formalism," The Journal of Chemical Physics, vol. 132, no. 11, Article ID 114110, 2010.

[57] A. V. Marenich, C. J. Cramer, and D. G. Truhlar, "Universal solvation model based on solute electron density and on a continuum model of the solvent defined by the bulk dielectric constant and atomic surface tensions," The Journal of Physical Chemistry B, vol. 113, no. 18, pp. 6378-6396, 2009.

[58] A.-R. Allouche, "Gabedita-a graphical user interface for computational chemistry softwares," Journal of Computational Chemistry, vol. 32, no. 1, pp. 174-182, 2011.

[59] P. Mori-Sánchez, A. J. Cohen, and W. Yang, "Localization and delocalization errors in density functional theory and implications for band-gap prediction," Physical Review Letters, vol. 100, no. 14, Article ID 146401, 2008.

[60] A. J. Cohen, P. Mori-Sánchez, and W. Yang, "Insights into current limitations of density functional theory," Science, vol. 321, no. 5890, pp. 792-794, 2008.

[61] D. Jacquemin, A. Femenias, H. Chermette et al., "Assessment of several hybrid DFT functionals for the evaluation of bond length alternation of increasingly long oligomers," Journal of Physical Chemistry A, vol. 110, no. 17, pp. 5952-5959, 2006.

[62] D. Jacquemin, E. A. Perpète, G. Scalmani, M. J. Frisch, R. Kobayashi, and C. Adamo, "Assessment of the efficiency of long-range corrected functionals for some properties of large compounds," The Journal of Chemical Physics, vol. 126, no. 14, Article ID 144105, 2007.

[63] S. Yang and M. Kertesz, "Bond length alternation and energy band gap of polyyne," The Journal of Physical Chemistry A, vol. 110, no. 31, pp. 9771-9774, 2006. 

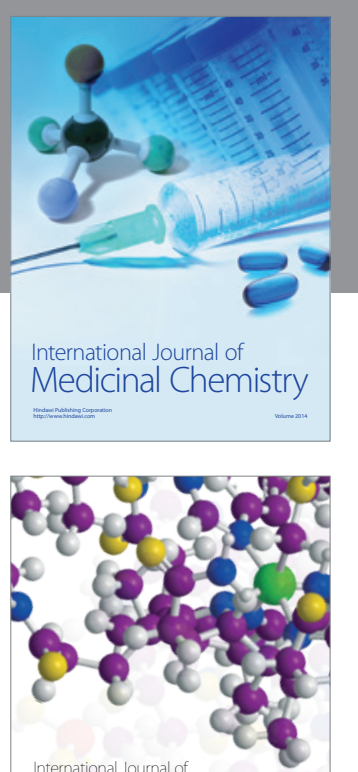

Carbohydrate Chemistry

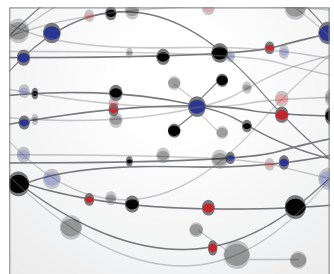

The Scientific World Journal
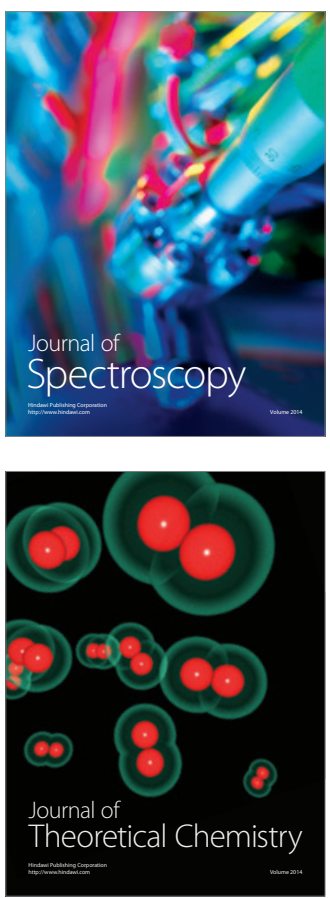
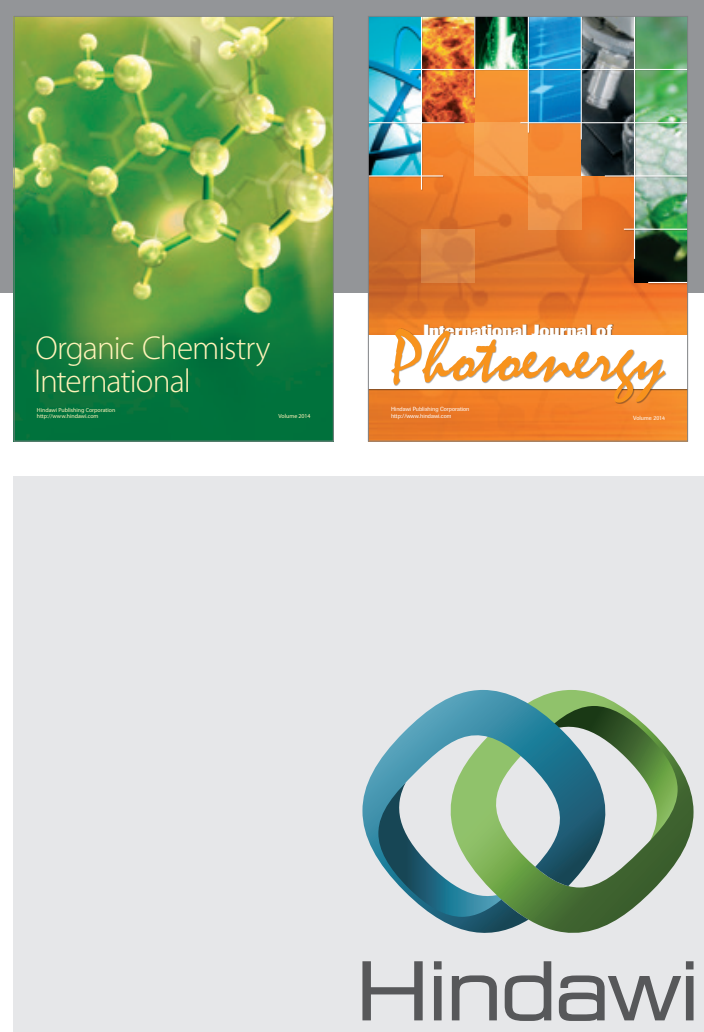

Submit your manuscripts at

http://www.hindawi.com

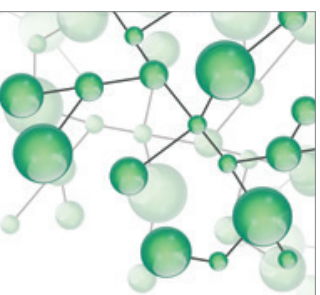

International Journal of

Inorganic Chemistry

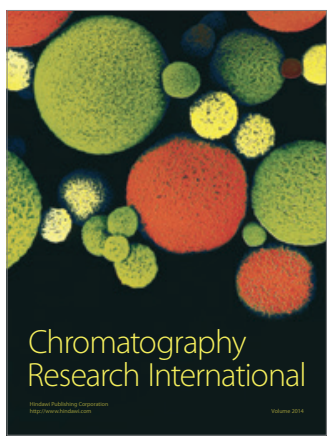

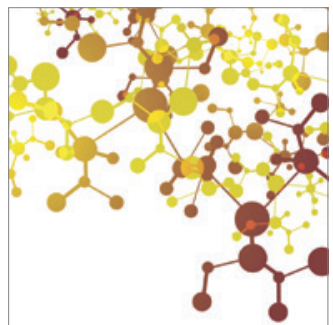

Applied Chemistry
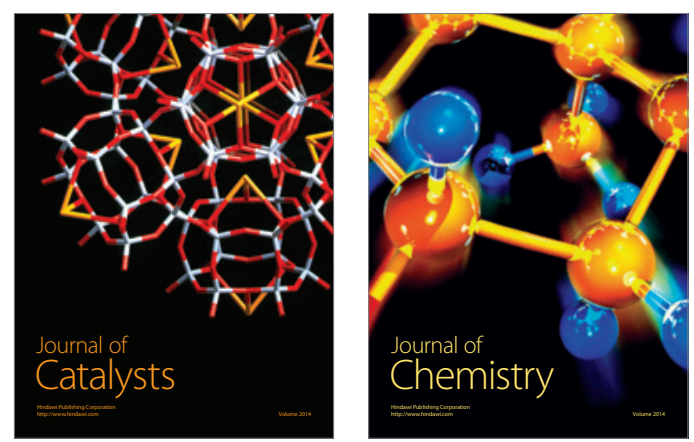
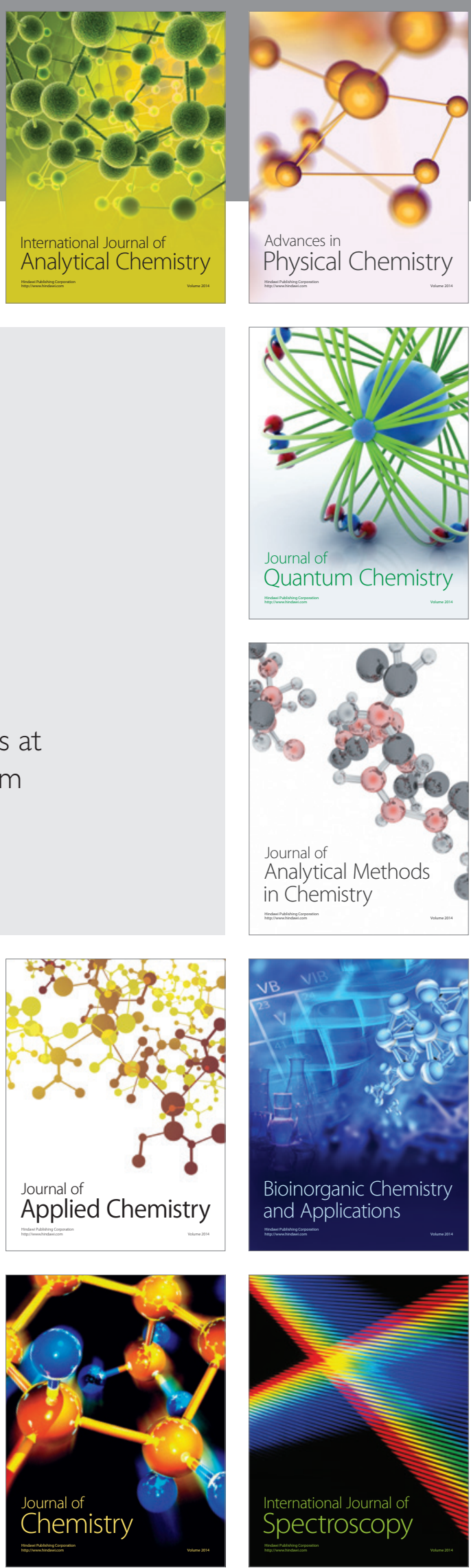